\title{
BRITISH DENTAL CONFERENCE AND EXHIBITION PREVIEW
}

Please send product news information and images to Kate Quinlan at the BDJ, Nature Publishing Group, The Macmillan Building, 4-6 Crinan Street, London, N1 9XW. Product news is provided as a service to readers using text and images from the manufacturer, supplier or distributor and does not imply endorsement by the BDJ. Normal and prudent research should be exercised before purchase or use of any product mentioned.

\section{INTRODUCING THE GOLD STANDARD}

Endodontic treatment has never been an easy undertaking. Referral to a specialist or extraction of the potentially treatable teeth involved is often preferred by GDPs, chiefly because of the fear of file separation.

However, the past decade has seen major advancements in file systems and the materials used in their construction to make endodontic treatment easier to perform for both endodontic specialists and general dentists alike.

DENTSPLY have been at the forefront of revolutionising endodontic treatment. The introduction of ProTaper ${ }^{\circledR}$ instruments in 2001 to the launch of the WaveOne ${ }^{\circledR}$ reciprocating single file endodontic system in 2011, has led to a real progression in superior root canal preparation procedures and patient safety.

DENTSPLY's WaveOne system saw the introduction of M-Wire NiTi technology, resulting in files that have become increasingly flexible with a marked resistance to cyclic fatigue (the leading cause of file separation).

Since the introduction of Wave0ne, DENTSPLY have continued to research the enhancement of the single file system, taking into account the increasing move towards minimally invasive endodontics and listening to the feedback from those clinicians using the system.

Working in conjunction with four of the international key opinion leaders involved in WaveOne's initial development, Dr Cliff Ruddle (USA), Dr Sergio Kuttler (USA), Dr Wilhelm Pertot (France) and Dr Julian Webber (UK), the aim has been to improve both the cutting efficiency and mechanical properties of the file, giving increased confidence to those still wary of carrying out endodontic procedures and those using the current system.

The result is the launch of an exciting new generation of reciprocating files - DENTSPLY's WaveOne GOLD. Keeping to the simplicity of the first generation

The WaveOne ${ }^{\circledR}$ GOLD NiTi files are part of a complete treatment solution including dedicated WaveOne GOLD paper points, variable tapered gutta-percha points which more closely match the shape created with the files, and THERMAFIL ${ }^{\circledR}$ obturators. WaveOne GOLD is also compatible with GuttaCore and the $\mathrm{X}$-Smart Plus Motor.
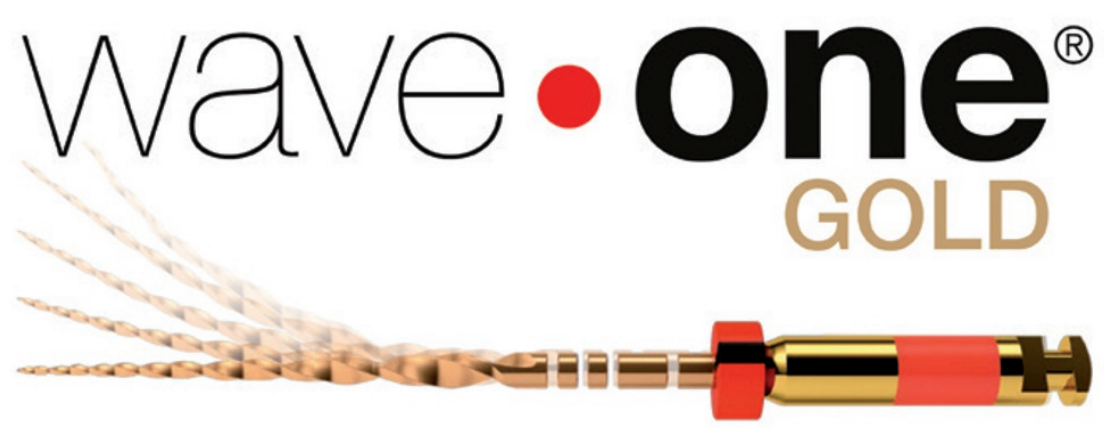

WaveOne system, WaveOne GOLD now offers strong additional benefits over its predecessor.

\section{Shaping canals has just got easier...}

The distinctive gold appearance of the WaveOne GOLD files is the result of a unique heat-treatment process applied after manufacture. The raw metal is nickel titanium which is repeatedly heated and cooled, giving it not only its gold colour, but also considerably improving its strength and flexibility.

The reciprocating WaveOne GOLD cross-section has been enhanced from the triangular shape of its predecessor to a unique parallelogram design that gives one or two cutting edges depending on the location along the file. These edges are designed to minimise the screwing effect on the canal walls greatly reducing torque, improving cutting efficiency and allowing better removal of debris. Cyclic fatigue resistance of WaveOne GOLD Primary has been improved by 50\% over WaveOne Primary and is now twice as resistant as most standard rotary systems, further reducing any risk of file separation and therefore enhancing patient safety.

The increased strength and flexibility allows for negotiation of a wider range of canal morphologies, helped by an increase in the range of file sizes now available - small \#20.07; primary \#25.07; medium \#35.06, and large \#45.05, in a choice of three lengths (21, 25 and $31 \mathrm{~mm}$ ). The majority of cases (around $80 \%$ ) can still be treated with the WaveOne GOLD Primary using just the one single file to start and complete the shaping of the canal.

With the introduction of WaveOne GOLD the emphasis has been to enhance the confidence of dentists. Dr Webber said: 'We believe that the enhancements we have made in WaveOne GOLD will increase the clinicians' confidence, help take away the fear factor and encourage them to take on cases considered too difficult in the past.' DENTSPLY on 08000723313 or visit dentsply.co.uk/gold. Earn DENTSPLY Rewards £s to spend on more of the products you love at dentsplyrewards.co.uk and access FREE CPD webinars and product demonstrations at dentsplyacademy.co.uk
For more information contact 